\title{
PRESSURIZED WATER REACTOR DEVELOPMENT, MECHANICAL-STRUCTURAL REQUIREMENTS AND PROBLEMS
}

\author{
W. KELLER \\ Siemens Aktiengesellschaft, Reakiortechnik, D 852 Erlangen, Federal Republic of Germany
}

Received 23 November 1971

\begin{abstract}
The present survey of pressurized water reactor development and the related mechanical-structural requirements and problems focuses on the following points: Development trends in power density, burn-up and fuel costs; mechanical problems of reactor vessel and internals; development trends in loop capacity and live steam pressure; method of operation and effectiveness of the safety injection and core emergency cooling systems; problems related to the containment structure.
\end{abstract}

\section{Introduction}

A practice has been adopted of differentiating between "conventional" and "advanced" reactors with the term conventional being apparently applied to those reactor designs which have been built in larger numbers and have thus proved the soundness of their engineering concept. One of the best known representatives of the conventional family is the pressurized water reactor (PWR). Its basic concept, which has not changed appreciably since the commissioning of YANKEE ROWE, the first commercial nuclear power station in 1960, is well known. The immense progress, however, made in pressurized water reactor engineering during the past decade seems to be overlooked sometimes.

For fully appreciating the advances achieved it must be recollected that the overall output of the 9 nuclear power stations with pressurized water reactors ordered up to the year 1964 was a mere $2600 \mathrm{MW}_{\mathrm{e}}$, at a mean unit output rating of $290 \mathrm{MW}_{\mathrm{e}}$. From 1964 to 1968 , a total of 43 nuclear power station orders was placed in the western part of the world alone, constituting a total of $32000 \mathrm{MW}_{\mathrm{e}}$, with the mean unit rating increasing to $740 \mathrm{MW}_{\mathrm{e}}$.

This growth continued irresistibly in the subsequent years up to the present. Orders for an additio- nal $38400 \mathrm{MW}_{\mathrm{e}}$ were signed with the unit rating averaging at about $900 \mathrm{MW}_{\mathrm{e}}$. The total generating capacity of nuclear power stations with pressurized water reactors under construction or in service is about $73000 \mathrm{MW}_{\mathrm{e}}$ for the western part of the world and a total of 112 plants with $85000 \mathrm{MW}_{\mathrm{e}}$ for the whole world. The largest units ordered so far will have an output rating of about $1300 \mathrm{MW}_{\mathrm{e}}$ net. These few data should help to convey an idea of the enormous engineering effort which was necessary and still continues to be required, particularly in the field of reactor component mechanical design, in order to attain the stated goals.

Progress in the past decade, however, was not limited to augmenting the output ratings alone. Numerous refinements in the design details of reactor components led to a high degree of standardization especially of their sensitive parts. This resulted in an appreciably higher operational reliability and consequently in a better plant availability. A steady reduction in fuel costs was achieved through a vast number of development steps in the fields of fuel cycling and fuel assembly design. These improvements were made possible by increasing the power density and burn-up and lowering parasitic absorption by the choice of Zircaloy cladding material instead of steel. Other contributing factors were the 
evolution of simpler ways of assembling fuel elements.

Improvements were also attained in the operational behaviour of pressurized water reactor power stations. With some of the new nuclear power stations it is now possible to use a major portion of their rated output for peaking duty whereas the first-generation plants were mainly designed for constant load duty. New reactor designs and modified control schemes permit fast and frequent load changes over a wide power range. Considerable improvements were achieved, last but not least, in the field of environmental safety.

This was not only realized by improving the efficiency of the containment systems - such as for instance by the introduction of new absorbent charcoal filters in the delay lines for fission gases - but also in the field of fault and accident prevention as well as by providing means of limiting the extent of damage in the case of accidents which, of course, cannot be ruled out entirely.

The first category encompasses the appreciably improved reactor protective systems and reactor com- ponents which are designed for easy maintenance inspections. The higher reliability and better redundancy of the safety injection and emergency heatremoval systems are typical examples in the second category. From this broad spectrum of development tasks a few items of particular interest will be discussed in the following pages.

\section{Development trends in power density, burn-up and fuel costs}

Starting with a brief repetition of the basic concept of the nuclear steam generating system in a pressurized water reactor fig. 1 shows a cross section through the containment structure of the BIBLIS nuclear power station. Characteristic for a pressurized water reactor is the separate primary loop, comprising the reactor pressure vessel (1), the steam generators (2), the main coolant pumps and the pressurizer which are not visible in this picture. The fuel pit (5) with storage racks for burned fuel

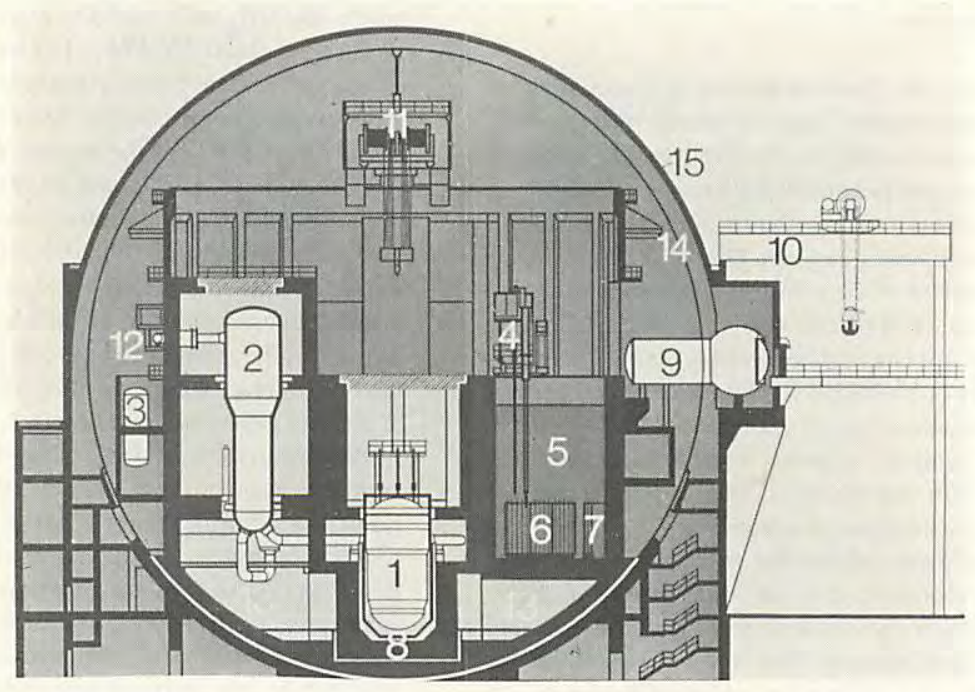

Fig. 1. Biblis nuclear power station reactor building. Legend: 1. Reactor pressure vessel; 2. Steam generator; 3 . Accumulator;

4. Refuelling machine; 5 . Spent fuel pit; 6 . Fuel storage rack; 7. Fuel-assembly; transfer station; 8 . Biological shield;

9. Equipment hatch; 10. Outward-transfer bridge; 11. Polar crane; 12. Main-steam lead; 13. Containment sump; 14. Steel containment shell; 15 . Concrete sheild. During operation accesible: dark grey, not accesible: light grey. 
assemblies (6) and new fuel assemblies (7) is located immediately adjacent to the reactor pit. Operational breaks for refuelling can be kept very short because of a refuelling machine (4), capable of traversing the short distance between both pits. The pressureretaining components of the primary loop are located within the pressure-tight containment shell (14) which is arranged inside a concrete structure providing additional shielding. The penetrations through this containment structure carry non-active media only, i.e. feedwater and live steam (12). The area shown light shaded beyond the secondary shield which also provides fragmentation protection can be entered after the reactor is shut down only, i.e. it is inaccessible during reactor duty. The areas shown dark shaded are accessible at all times.

One of the most important development objectives is, of course, the reactor proper with efforts aimed at obtaining the desired output with the smallest volume possible. Ways are sought to minimize the dimensions and weight of the pressure vessels, core internals and fuel assemblies and thus to cut the initial cost investment. Any reduction of vessel size is accompanied by a reduction of the water volume inside the pressure vessel. Any increase in power density thus obtained results in additional cost savings, since the containment design pressure can be lowered in proportion with the reactor water volume.

This development objective, however, imposes great demands on the mechanical design of the core proper. In fig. 2 a typical PWR fuel assembly of present design with inserted spider-type control assembly is shown. The progress achieved is illustrated by fig. 3 . Here the power density, the desired mean final burnup and the resultant fuel costs are plotted as a function of time, i.e. of the year in which the plant was ordered. Constant fuel fabrication costs at DM 400,per $\mathrm{kg}$ of uranium are assumed.

The mean power density has been increased continuously during the past ten years and this tendency should continue during the years to come as shown by that part of the curve which is drawn with interrupted lines. This, however, can be achieved only, if the mean burn-up is also raised. A further increase in the power density would not offer significant economic advantages, if the mean burn-up is permitted to remain constant at the present customary value of 31000 to $32000 \mathrm{MWd} / \mathrm{t} \mathrm{U}$. Gradually decreasing fuel costs,

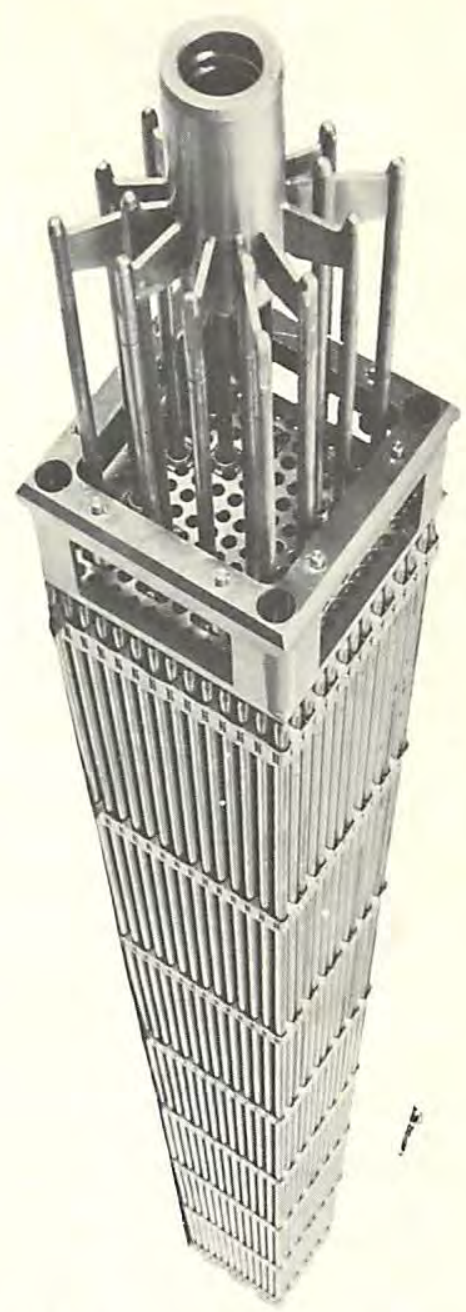

Fig. 2. Fuel element of the PWR at Obrigheim.

however, are attainable with increasing power density and mean burn-up parameters as shown in the graph, as is evidenced by the dotted part of the curves.

The design burn-up was raised to $31000 \mathrm{MWd} / \mathrm{t}$ between the years of 1963 and 1967, after preliminary data were available on single fuel rods. From then on it remained at the same level. This stagna- 


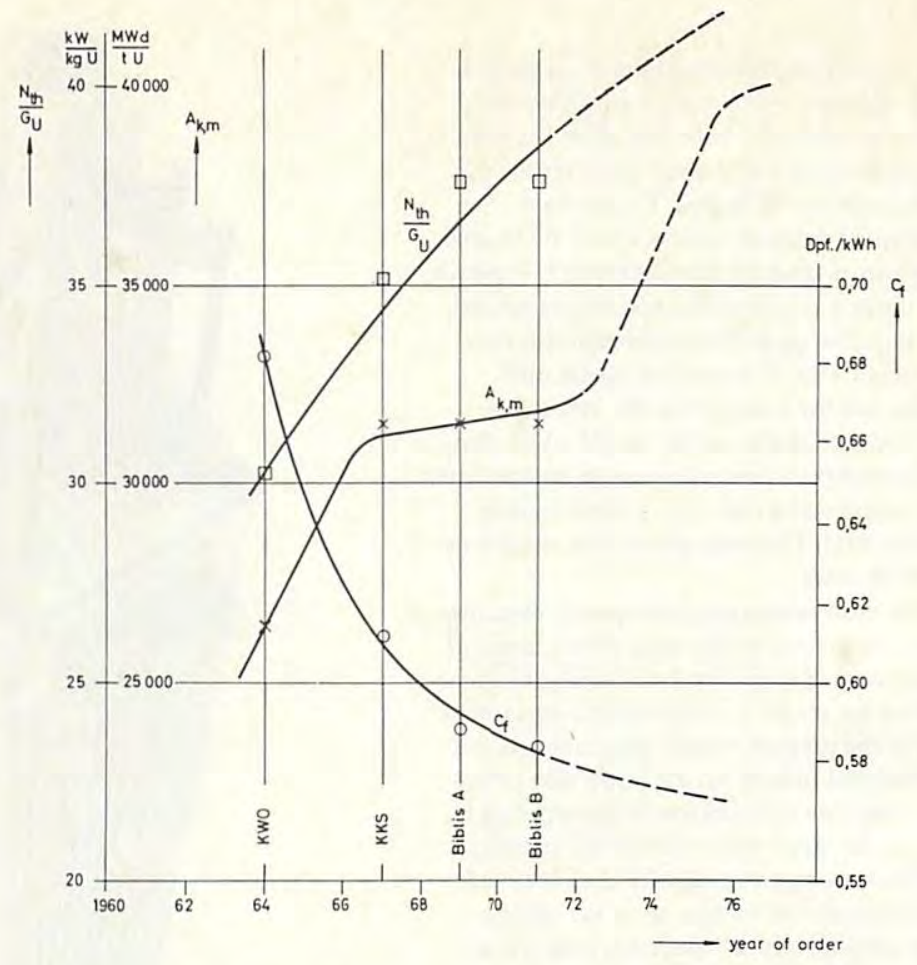

Fig. 3. Power density, fuel discharge burn-up and fuel cycle costs of PWR.

tion can be attributed to two main causes:

1. The economic incentive for a further increase in the mean burn-up is not very large, as can be seen by the flattening out of the fuel cost curve as the burn-up is raised.

2. The limited number of burn-up experiments conducted so far did not make it seem advisable to raise the burn-up level any further for the time being, particularly because the major portion of these tests were performed under conditions which cannot be regarded very representative.

Adequate irradiation data, however, should become available in 1972 for example with information flowing in from the fuel assemblies of the OBRIGHEIM nuclear power station. These should permit a further step to a burn-up level of about $40000 \mathrm{MWd} / \mathrm{t}$ being envisioned. This would offer still a further fuel cost reduction of $0.02 \mathrm{Pf}$ per kWh approximately.

The mechanical problems involved are illustrated in a simplified way in fig. 4 . The graph shows a plot of the equivalent strain of zircaloy -4 fuel cladding material versus the maximum local burn-up.

The dotted line illustrates the conditions as obtained in the first OBRIGHEIM core. Initially, the curve takes a steep dip into the negative range of the equivalent strain, i.e. the fuel cladding creeps under the external pressure of the coolant until it comes into contact with the fuel. This is followed by a short flat part bridging the elasticity range of the elongation. Afterwards, the curve rises into the positive quadrant owing to the swelling rate of the fuel. The swelling rate starts to increase at a value of 


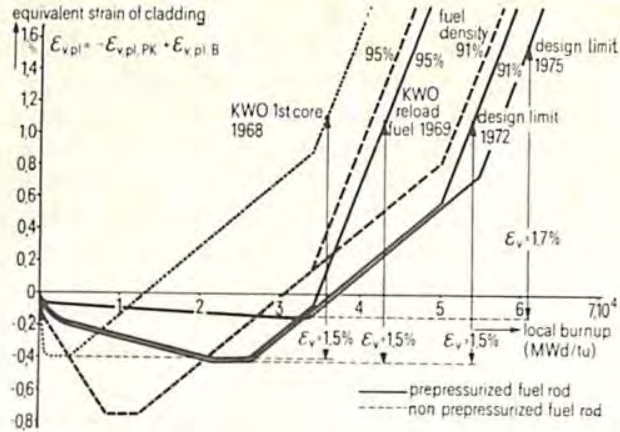

Fig. 4. Cladding deformation in a PWR fuel rod due to creep of cladding and swelling of fuel.

about $32000 \mathrm{MWd} / \mathrm{t}$ causing the break in this part of the curve. The allowable positive uniform equivalent strain is considered to be the most important design criterion. A maximum local burn-up of about $36000 \mathrm{MWd} / \mathrm{t}$ had to be foreseen with the initial OBRIGHEIM core in order to attain the quaranteed value of the mean burn-up. From the curve it can be seen that such a burn-up corresponds to a uniform equivalent strain of $1.5 \%$, a value which can be regarded safe at PWR canning temperatures on the basis of data available from numerous incore and outcore experiments. The maximum local burn-up achieved successfully at OBRIGHEIM so far is already $32000 \mathrm{MWd} / \mathrm{t}$ corresponding to a calculated equivalent strain of about $1.3 \%$

The second OBRIGHEIM fuel charge which was inserted in 1970 included the following three essential improvements:

1. Using canning tubes of zircaloy-4 with a dinstinct texture and hardness it was possible to reduce the creep rate under the given conditions. This offered a longer time interval until the cladding gets in to full contact with the fuel.

2. Internally pressurized fuel rods were employed for compensating the major part of the pressure differential existing between the coolant volume and the fission gas volume. This also reduced the creep of the cladding to a great extent.

3. The size of the gap between fuel and cladding was increased, thus permitting the fuel to swell over longer periods of time without contacting the fuel cladding.

These are the reasons why the reload curve shows an appreciably flatter initial dip and why the upward break does not occur until a higher burn-up of about $43000 \mathrm{MWd} / \mathrm{t}$ can be achieved, assuming a maximum uniform equivalent strain of $1.5 \%$ is safe for this type of cladding. The resultant mean burn-up then can be raised to somewhat more than 31000 $\mathrm{MWd} / \mathrm{t}$.

An additional step was taken at STADE nuclear power station. For the fuel elements which will reach a burn-up $\geq 30000 \mathrm{MWd} / \mathrm{t}$ the fuel density was lowered. For the original OBRIGHEIM fuel a density of $95 \%$ of the theoretical was chosen whereas for STADE the density in the high burn-up fuel was reduced to $91 \%$. The higher pore volume in the low density fuel gives more room for swelling with. in the fuel pellets. In this way it is possible to shift the point of transition from the lower to the higher swelling rate into a higher burn-up regime. A maximum permissible local burn-up of $54000 \mathrm{MWd} / \mathrm{t}$ can be achieved thus, if again a maximum uniform equivalent strain of $1.5 \%$ is deemed acceptable. This should make mean discharge burn-up levels of about $38000 \mathrm{MWd} / \mathrm{t}$ possible. The discharge burn-up leve's of $32000 \mathrm{MWd} / \mathrm{t}$, as guaranteed for STADE. have thus proved to be possible with margin, taking into account the new fuel assembly design and the experience accumulated with the OBRIGHEIM fuel assemblies regarding the equivalent strain.

We surely can state that the development in this direction has not yet reached its climax. The design limit 1975 shown in fig. 4 bases on the assumption that it will be possible to reduce the pressure differential between the fission gas volume and the coolant further while keeping this differential about constant until the end of life of any rod and to raise the permissible uniform equivalent strain to a value of at least $1.7 \%$ by developing canning tubes with better local in-pile ductilities. Investigations conducted in this field already returned promising results indicating that the stated values can he attained.

This would permit to realize maximum local burn-up levels of above $60000 \mathrm{MWd} / \mathrm{t}$ and mean burn-up levels of better than $40000 \mathrm{MWd} / \mathrm{t}$. A more detailed study of this complex will reveal other in- 
fluences as they are caused, for instance, by frequent load changes and fuel reshuffling procedures which must be taken into account. These, however, do not drastically change the situation described. The predictions regarding the development trends of power density, burn-up and fuel costs as discussed in fig. 4 may be considered as fairly conservative.

\section{Mechanical problems of reactor vessel and internals}

Step increases in the unit power ratings were accom. panied by a wide variety of development problems. One of these is the fabrication of the reactor pressure vessel. This evolution is presented in fig. 5 . The pressure vessel for the OBRIGHEIM nuclear power station (upper left) has an overall weight of $195 \mathrm{t}$ with an inner diameter of $3.27 \mathrm{~m}$ in its cylindric portion. The pressure vessel for the BIBLIS nuclear power station (lower right) on the other hand weighs $450 \mathrm{t}$ at an inner diameter of about $5 \mathrm{~m}$. The deviation from the proportionality between pressure-vessel weight and reactor output can be attributed to an increase in power density and better material utilization.

While the first pressurized water reactors have had a power density of $27 \mathrm{~kW}_{\mathrm{th}} / \mathrm{l}$, the OBRIGHEIM reactor already offered a $77 \mathrm{~kW}_{\mathrm{th}} / 1$ ratio and BIBLIS, finally, has a power density of about $100 \mathrm{~kW}_{\mathrm{th}} / 1$. Expressed more drastically, it can be stated that 3500 MW of thermal energy are generated in the extremely small space of $4 \times 3 \times 3 \mathrm{~m}^{3}$. This high power density is one of the decisive reasons why the light-water moderated reactor shows off so well against its competitors in the field of initial costs.

Advances were not limited to the overall dimensions - the maximum wall thickness and unit weights are given in the upper right of fig. 5 - but were also attained in the fields of design, fabrication and, primarly, testing. In this context special mention must be made of new disclosures in the field of mechanics of fractures. Today rather reliable predictions on the critical lengths of fractures can be made, if stress, neutron dose, wall thickness and temperature are known. (Critical lengths are those lengths of fractures at which an instantaneous brittle fracture will occur). In addition, it is also possible to calculate the crack propagation rate for small fractures as a function of the number and amplitude of changes in stress.

Typical values of crack propagation in a $1200 \mathrm{MW}_{\mathrm{e}}$

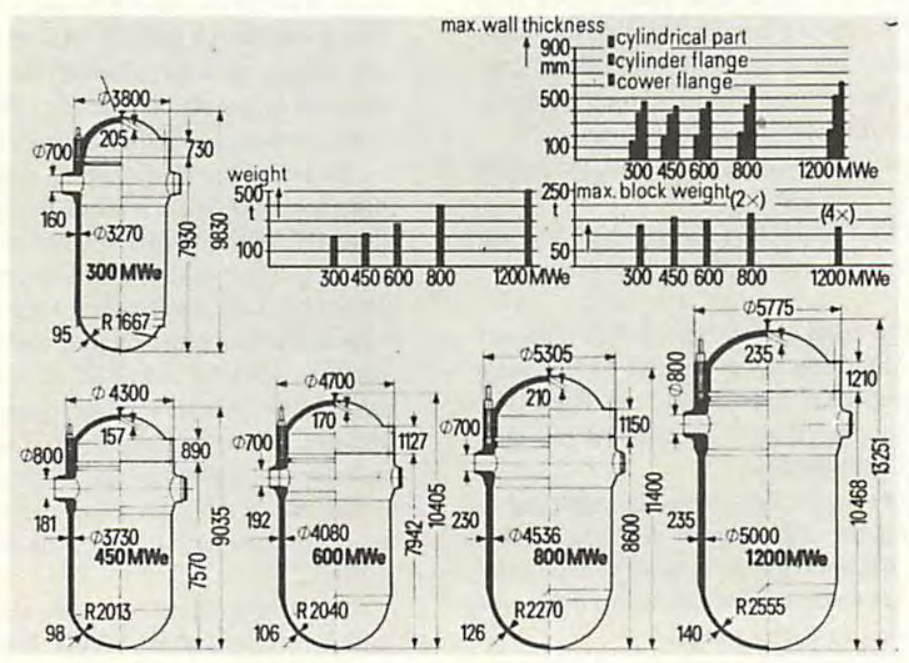

Fig. 5. Pressurized water reactor pressure vessels. 
reactor pressure vessel are given in fig. 6. On the ordinate axis the depth of hypothetically assumed semicircular cracks is plotted as a function of the number of years of operation. The lowest curve (1) defines the smallest defect which can be detected by ultrasonic devices having a dimension of about $4 \mathrm{~mm}$. Its growth rate is hardly discernible in the curve and consequently was stated as being $0.06 \mathrm{~mm}$ within 40 service years in the column to the right of the chart. This value bases on an assumed number of 240 cold shutdowns during the period studied.

Curve (2) defines a defect which - according to present practice - is considered acceptable and needs not necessarily be removed. This defect with an initial dimension of $20 \mathrm{~mm}$ will grow by $0.4 \mathrm{~mm}$ only within 40 years of service. In curve (3) a defect of $76 \mathrm{~mm}$ is plotted. With such a defect the $0.2 \%$ yield strength of the material would just be reached. Even such a defect which certainly would be detected by ultrasonic or X-ray equipment would grow by $3.5 \mathrm{~mm}$ only

Crack geometry $a / 2 \cdot c=0,5$

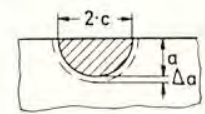

Pressure vessel data

\begin{tabular}{|l|l|c|c|}
\hline Material & - & $22 \mathrm{NiMoCr} 37$ & - \\
Wall thickness & $\mathrm{s}$ & 220 & $\mathrm{~mm}$ \\
Integrated neutron flux & $\phi \cdot t$ & $5 \cdot 1018$ & $\mathrm{nvt}$ \\
Yield strength & $\sigma_{\mathrm{a} 2}$ & 400 & $\mathrm{~N} / \mathrm{mm}^{2}$ \\
Stress (pressure test) & $\sigma$ & 260 & $\mathrm{~N} / \mathrm{mm}^{2}$ \\
Stress (operation) & $\sigma$ & 178 & $\mathrm{~N} / \mathrm{mm}^{2}$ \\
Cycles (heat up,cool down) & $\mathrm{N}$ & 240 & $\mathrm{LW}$ \\
Stress range & $\Delta \sigma$ & 400 & $\mathrm{~N} / \mathrm{mm}^{2}$ \\
Crack depth/crack length & $\mathrm{a} / 2 \mathrm{c}$ & 0.5 & - \\
\hline
\end{tabular}

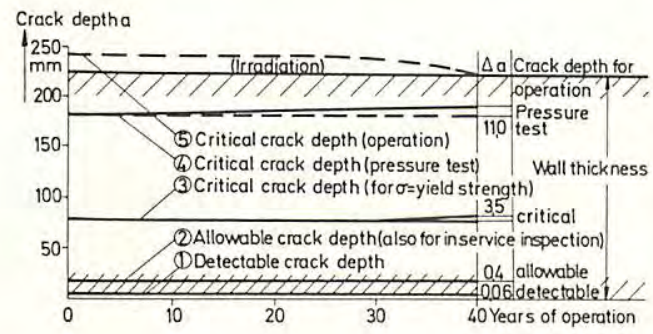

Fig. 6. Crack growth $\Delta a$ for reactor pressure vessel. within the period under consideration und thus would fail to pose any real danger.

Curve (4) denotes a fracture dimension which would result in a brittle fracture during a cold pressure test, i.e. a pressure test conducted at room temperature. This means that any crack larger than the dimension plotted for curve (4) would automatically result in a rupture of the pressure vessel during the cold pressure test. At operating temperatures the critical length of fractures is appreciably higher, as evidenced by curve (5). It is, in effect, higher than the overall wall thickness of $220 \mathrm{~mm}$ shown in fig. 6 over the whole range of operating parameters.

This means that the leak-before-break criterion is met for all operating conditions. In other words, water would leak out of a throughgoing fracture in the pressure vessel into an area which is being monitored for such leakages before any brittle fracture can occur. The aforesaid consequently allows the following conclusions:

1. All possibly existing defects, down to sizes far below the critical limits, can be clearly located with the methods presently available.

2. Should such faults remain undetected in spite of the most careful testing, they will be noted during the pressure test at room temperature and thus will be prevented from reaching critical values at operating temperatures.

3. The fracture propagation rate is so slow that any defects which possibly remained unnoticed during the non-destructive testing cannot reach critical dimensions during the assumed service life. This knowledge of the mechanics of fractures used in conjunction with the highly sophisticated methods secures that the possibility of a rupture of the pressure vessel can be ruled out. The same also applies to the relative short connecting pipes between steam generators, main coolant pumps and the pressure vessel which must grow in diameter as the unit ratings are increased. For instance, the main coolant pipe diameter for a 4-loop plant with an output of $1200 \mathrm{MW}_{\mathrm{e}}$ is $0.8 \mathrm{~m}$. Such connections can no longer be considered as pipelines in the original sense of the word. Rather, they are part of the closed primary loop and are - at least in some power stations of the same material and designed, specified, fabricated and tested in the same manner as the pressure 
vessel. Thermal gradients and vibration loads in these connections are kept extremely low and are fully reflected in the stress analysis. Consequently, the same applies to the probability of a pipe rupture as had been said for the pressure vessel, i.e. that a brittle fracture is out of question at the present state of the art in the design and fabrication of reactor parts.

Such recognition of the advanced knowledge available should go in hand with a simplified plant safety concept. Unfortunately, this is not yet the case because of the historical background of the reactor development. Mainstay of all reactor safety engineering considerations is still the possibility of a double-ended instantaneous fracture of one main coolant pipe.

This safe ty philosophy necessitates extensive safety precautions at different points, particularly as the loop capacities are increasing. It is required, for instance, that the core residual heat removal capability shall be retained following a postulated main pipe fracture at any point of the system. This means the reactor core must not undergo any appreciable deformation which might endanger post accident cooling of the core. This far reaching in tegrity of the core structure and the fuel elements only can be proved by rather complex analytical studies. Any pipe fracture would result in shock loads of the core structure and induce vibrations. Their control has a decisive influence on the design of the individual components. Other phenomena influencing the mechanical design of the core internals to be mentioned in this context are the vibration loads induced by local and bulk pressure fluctuations with various frequencies during normal operation conditions.

In fig. 7 intensity and frequency of oscillation inducing phenomena which were measured in the primary loop of a $600-\mathrm{MW}$ reactor are plotted in a doublelogarithmic scale. In the lower left there is a shaded area denoting the $3-$ to $30-\mathrm{Hz}$ band. Vortex shedding at different locations of the core internals is mainly responsible for excitations in the low-frequency band. Resonances are not established by these excitations because the shedding frequencies are below the fundamental natural frequencies designed into all the core internals. Pronounced peaks were observed at $10 \mathrm{~Hz}$ and $28 \mathrm{~Hz}$ only. The first of these frequencies could be correlated with random pendulum vibrations of the core vessel and pressure vessel. Consequently care must be taken to see that none of the core internal compo- nents, e.g. control rods, control rod guides, instrumentation probes, fuel assemblies, etc. have natural frequencies in that width of the spectrum.

Outside of this range there are quite a number of discrete excitation frequencies, such as for instance either the multiple of the pump speed denoted $F 1$ to $F 4$, or the multiple of the product of pump speed and number of impeller blades, denoted $f 1$ to $f 12$. In general it can be said that the intensities of the higher frequencies have only a negligible influence on the design of the internals. In the case on hand special consideration had to be given to the excitation frequencies of $75 \mathrm{~Hz}$ and $150 \mathrm{~Hz}$.

It should be mentioned, however, that in a special case trouble was caused by a relatively high excitation frequency of $600 \mathrm{~Hz}$ which was capable of destroying an irradiation channel during trial operations. Parts of the channel walls were induced to vibrate with a natural frequency of very close to $600 \mathrm{~Hz}$. Resonances resulted in an overstressing of the material. The channel had to be removed and replaced by one tuned to a different frequency. Fig. 7 should demonstrate that it is generally necessary but also possible to adjust the inherent frequencies of all core internals in order to prevent inadmissible oscillations at all locations.

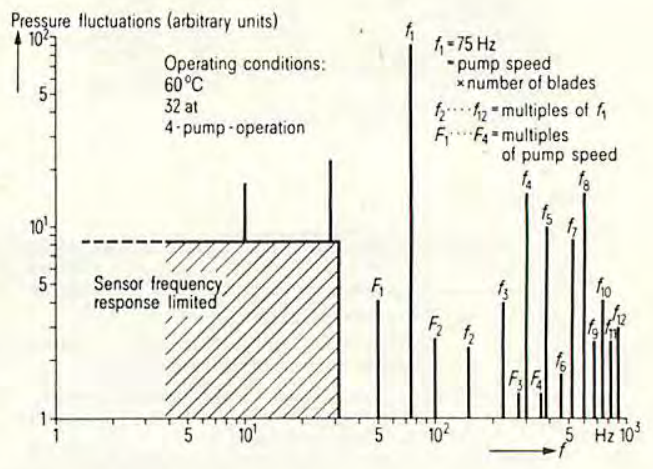

Fig. 7. Excitation frequencies in the main coolant circuit of a $600-\mathrm{MW}_{\mathrm{e}}$ PWR 


\section{Development trends in loop capacity and live steam pressure}

Aiming at ever increasing loop capacities is the economically sound consequence of raising the unit output ratings of pressurized water reactors. In the upper chart of fig. 8 the growth of the loop capacity is presented as a function of the year in which the reactor was commissioned.

The first pressurized water reactors had loop outputs of about $200 \mathrm{MW}_{\text {th }}$ while the new $1200 \mathrm{MW}$ power stations scheduled for commissioning in the years from 1973 to 1977 will have loop capacities ranging from 800 to $1800 \mathrm{MW}_{\text {th }}$, depending on whether four-, three- or two-loop designs are chosen. We tend to favour the four-loop alternative at this power level because of safety considerations.

The lower chart of fig. 8 shows the advance in live steam pressure. The first plants operated at a live steam pressure of below $40 \mathrm{kgf} / \mathrm{cm}^{2}$ which was increased gradually during the course of the years to a value of $60 \mathrm{kgf} / \mathrm{cm}^{2}$ customary for the plants presently on order. It is quite probable that the live steam pressure will be raised to values around $75 \mathrm{kgf} / \mathrm{cm}^{2}$ during the period under consideration, that is up to 1980. This higher steam pressure can be achieved by raising the temperature level in the primary loop on one hand and by increasing the heat transferred per unit surface on the other.

This augmentation of the live steam pressure was accompanied by an improvement in plant efficiency. Initially, efficiencies of $30 \%$ were acceptable; step by step they were brought up to values of $33 \%$ and $34 \%$. In the individual cases these values depend largely on the proporties of the cooling water available. The improved efficiency values do not only have a favourable effect on the specific initial costs, they also tend to reduce the running fuel costs which are inversely proportional to the degree of efficiency.

The specific problem in the development of the steam generators is the goal of obtaining high volumetric rating in order to keep the volume of the steam generators as low as possible. This requires particular care in the design of the steam separators which, at high throughput rates, tend to instabilities and a loss of their separation capacity. Manipulating
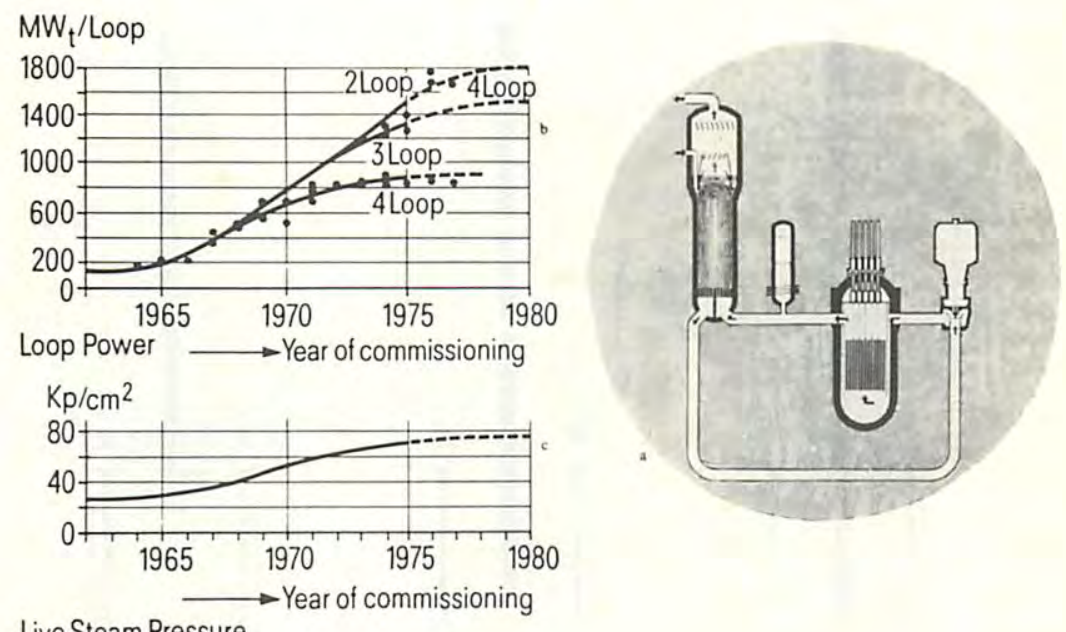

Live Steam Pressure

Fig. 8. (a) Pressurized water reactor, (b) loop power and (c) live steam pressure. Legend: 1 . Reactor; 2. Steam generator; 3. Main coolant pump; 4. Pressurizer. 
openings with remote-controlled devices are provided at the lower dome which permit to locate and eliminate any possible leaks at the tube plate as quickly as possible. Plugging defective tubes by explosive welding has proved to be very successfull. In this manner it is possible to reduce the risk of a steam generator leak to an acceptable minimum.

Easy access for repairs and inspections is a requirement that exists not only for the steam generators but also for the reactor proper. The new design include provisions permitting the inservice testing of all core internals and of the reactor pressure vessel at minimum time requirement. This is demonstrated by fig. 9 .

All pressure-vessel closure-head nuts can be opened practically simultaneously with a hydraulic spanning device. Thereafter, the closure head including the control rod drives can be lifted off with one lift of the reactor building crane. Concurrently the pit above the reactor is flooded with borated water. Another hoist is available for removing the upper core internals, thus making the reactor ready for the removal of the fuel assemblies. After the 121 to 193 fuel assemblies have been removed, the entire core barrel also can be lifted out with one lift. All these actions described should not take longer than about two days. The core internals are deposited under a shield of water and thus are accessible for visual inspections.

Subsequently, the entire inner surface is accessible for inspection and can be checked with optical devices, TV cameras or ultrasonic test equipment. The measuring instruments are mounted on a spider leg that can be pivoted on a rotable boom. A mechanism is provided for quickly scanning the entire surface of the pressure vessel as well as the inner faces of the main coolant inlet and outlet nozzles. There is no doubt that these facilities greatly contribute to the operational reliability and safety of the plant.

\section{Method of operation and effectiveness of the safe- ty injection and core emergency cooling systems.}

All PWR safety considerations focus on the possibility of a loss of core cooling capacity owing to damage to the reactor core.

The requirement for providing evidence that all possiblities of a primary circuit rupture in the pressurized water reactor have to be taken into account

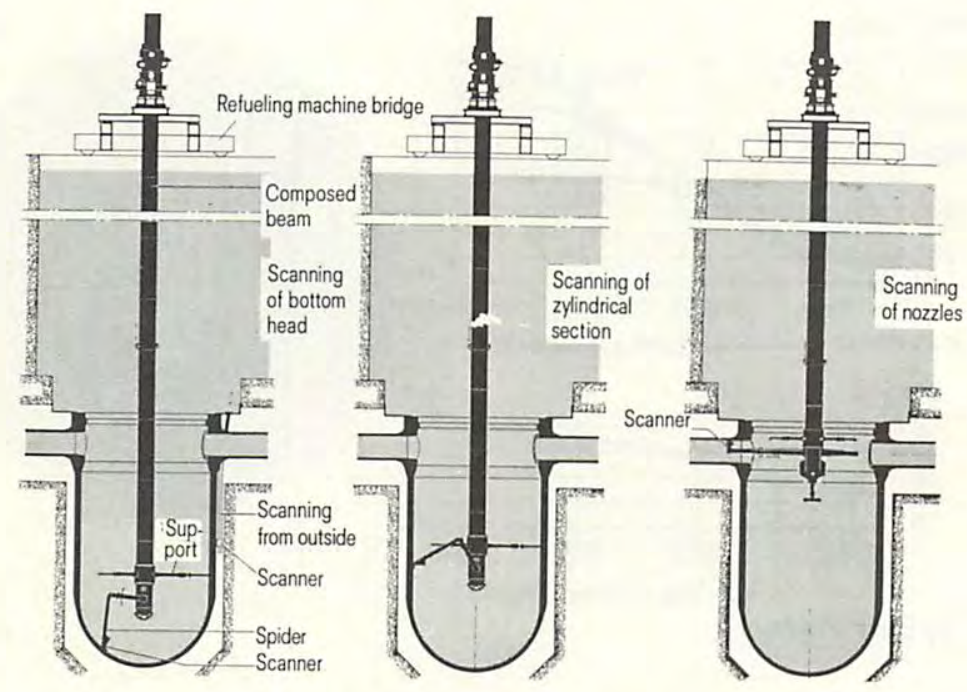

Fig. 9. Ultrasonic in-service inspection on pressurized water reactors. 
is being uphold by the Licensing Authorities in spite of the improbability of such a pipe rupture explained above. Design requirements differentiate between two categories of feasible faults: medium-sized leaks as they are caused by ruptures of pipes with nominal widths of up to $200 \mathrm{~mm}$, and limiting design faults such as the double-ended rupture of a main coolant line.

In a four-loop $1200 \mathrm{MW}$ plant the limiting design case thus can be postulated as the double-ended rupture of a pipe having a clearance width of $800 \mathrm{~mm}$. Within $15 \mathrm{sec}$ after such a fault the primary circuit would be completely drained and the primary system pressure would equalize with the pressure inside the containment structure. Instant availability of coolant at a high feed rate and relatively low head therefore is required for accomodating the limiting design case whereas a high-pressure lowrate system is needed for compensating the loss of coolant through a medium. sized leak. Dictated by the different fault conditions feasible, a core emergency cooling system was developed which comprises a high-pressure injection system coping with the medium-sized leaks as well as the high-rate accumulators and a low-pressure system for sustained core emergency cooling in the case of limiting design faults.

Doubts regarding the effectiveness of such core emergency cooling concepts were raised some time ago following tests conducted at IDAHO FALLS. But a more detailed description of the safety injection system designed for the SIEMENS pressurized water reactors proves this skepticism as not justified. Fig. 10 shows the basic design of the safety injection system which is provided in all the new four-loop plants. For reasons of redundancy the system consists of four parallel and completely separate trunks. The high-pressure injection system, the accumulators for the low-pressure system and the standby diesel generator of each trunk form one functional unit and are assigned to one specific primary loop.

The high-pressure injection system (4) is designed for keeping the core constantly flooded, even in the case of a rupture of the largest pipe connected to the reactor coolant system. Damage to the fuel assem. blies due to inadequate cooling is thus reliably prevented.

Core re-wetting in the case of maximum design leakages is guaranteed already before the end of the

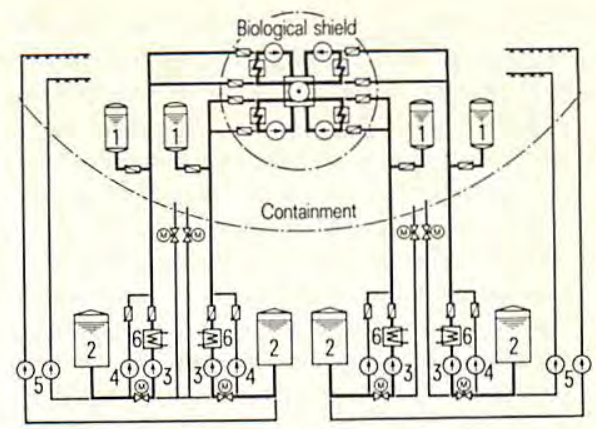

Fig. 10. Safety injection system of Siemens pressurized water reactors. Legend: 1 . Accumulator; 2 . Borated water storage;

3. LP-feed pump; 4. HP-feed pump; 5. Spray pump.

blowdown period by the accumulators which stand under a constant gas pressure of about $30 \mathrm{kgf} / \mathrm{cm}^{2}$.

After the deluge from the accumulators sustained core cooling is provided by the low-pressure system (3).

A look at the details of one discrete trunk will show that injection into the associated primary loop is effected through two separate injection lines per trunk, one entering the hot side and the other the cold side of the primary loop between the steam generator and the main coolant pump.

Activated in the case of a maximum design accident, consequently the accumulators feed through two equivalent injection lines. Check values automatically isolate the accumulator from the overall system when its gas pressure drops below the head of the residual heat removal pump which has been started up while the accumulator drops its volume of borated water. In the low-pressure mode the residual heat removal pumps (3) deliver cold, borated water from the borated water flooding tank (2) through double valves that need not be activated in the case of an accident since they are kept open during normal duty, into the reactor.

After 30 minutes of residual heat removal operation the borated water reserve is exhausted; the double valves are closed and the low-pressure system is switched over to squeeze recirculation cooling by opening up the appropiate pump valves and connecting the residual heat exchangers into the circuit.

Safety injection in the case of medium-sized leakages must overcome the initially high primary- 
system pressure. This is achieved with the high-head safety injection pumps which also draw from the borated water flooding tank. An automatic hydraulic control system (not shown in fig. 10) prevents the waste of borated water, if a safety injection line should burst.

The delivery rates of the residual heat removal pumps and of the high-pressure safety injection pumps of two of the four system trunks are sufficient to provide adequate core emergency cooling. Added redundancy is provided by enabling one injection line of the low-pressure system to feed into the ruptured primary loop. The accumulator overall volume is adequate to allow for the complete failure of one single accumulator and of one injection line and for one half of the volume of a second accumulator to flow out through the primary system leak.

Power supply for the core emergency cooling system is provided by 4 standby diesel generating sets, one assigned to each trunk unit. This redundancy is augmented by the normal power supply system.

Practically free of active parts, the pressure accumulator system has a failure probability of only $10^{-6}$ failures per demand. The failure probability of the highand low-pressure pump systems, if they are tested monthly, is $5 \times 10^{-5}$ failures per demand.

Analyzing the design or calculating probabilities, the conclusion will remain the same: a failure of this highly redundant core emergency cooling system is practically out of question.

In fig. 11 the effectiveness of the core emergency cooling system is demonstrated for the case of a double-ended main coolant pipe rupture and the effects of such an accident on the fuel assembly in the hot channel are analysed. Chronologically, the accident can be broken down in to two distinct phases, the depressurization (= blowdown) phase and the flooding phase.

As shown in both charts, the blowdown covers a time period of about $15 \mathrm{sec}$. During this time the pressure in the primary system drops from about $160 \mathrm{kgf} /$ $\mathrm{cm}^{2}$ to a uniform value of roughly $5 \mathrm{kgf} / \mathrm{cm}^{2}$ in the containment. The accumulators start feeding borated water into the core shortly before the depressurization phase is terminated. During the initial blowdown phase the coolant flow through the core stagnates for a short period of time approximately three seconds after the accident, causing the steam content to rise steeply. It, however, will drop back to about $30 \%$ as the circulation picks up again. The temperature of the fuel cladding in the hot channel will quickly rise to about $700^{\circ} \mathrm{C}$ during the brief stagnation of cooling-water flow, owing to the impaired heat transfer caused by the high steam content. In this postulated case it is assumed that transition boiling sets in $1.5 \mathrm{sec}$ after the accident. As the coolant circulation picks up again, the heat transfer is greatly improved. Gradually, the stored thermal energy is removed and the cladding and mean fuel temperatures decrease in the final phase of the blowdown. This can be seen in the upper chart of fig. 11, in which the cladding temperatures are plotted for the core midplane, the lower and the upper edge of the core. Also shown is the mean fuel temperature for the core midplane.

In the lower chart of fig. 11 the water level prior to and during the flooding phase is shown. Coolant, dumped by the accumulators, reaches the lower boundary of the core 25 seconds after the pipe rupture and rewetting of the fuel rods commences. With the fuel rods inadequately cooled during the preceding period of transition, their cladding temperatures had approached the mean fuel temperature. Thus, rewetting of the hot fuel rods starts a violent formation of steam, accompanied by an upward surge of water droplets. This moisture cooling immediately limits the temperature in the upper part of the core which is not yet flooded and initiates a uniform reduction of the temperature. With the water steadily rising in the core, moisture cooling is followed by nucleate boiling, resulting in an immediate temperature drop. When the cladding temperature falls below saturation temperature nucleate boiling will be replaced by single phase convective heat transfer.

For a detailed determination of the transient temperature, pressure, flow distribution and steam content during the blowdown process, the primary system was subdivided into 15 nodes. These investigations disclosed that the flow distribution within the primary system and the resulting heat transfer coefficients to be assumed during the blowdown phase and the flooding phase are of primary signifance.

Tests for the experimental clarification and confirmation of these phenomena were conducted in the Federal Republic of Germany and in the United States independently. Test results obtained in America and by SIEMENS and EURATOM in Europe confirmed our analy tical assumptions. 


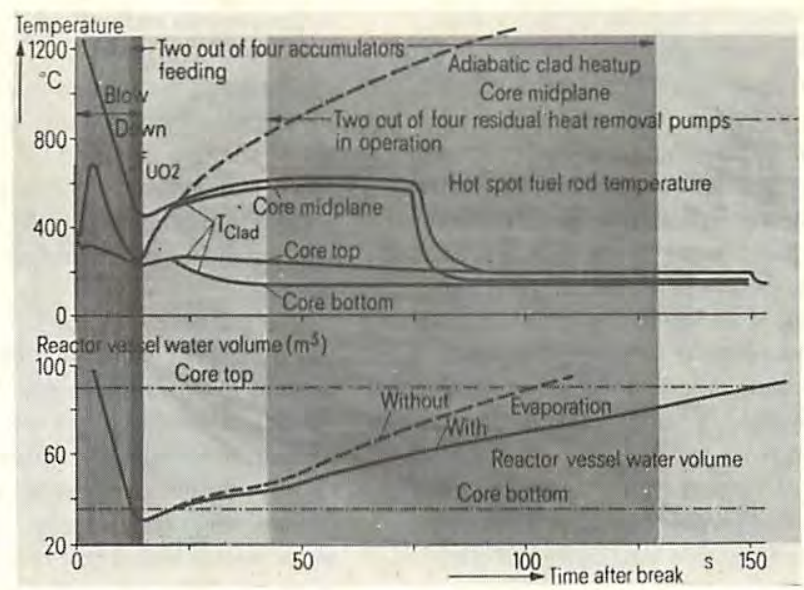

Fig. 11. Siemens $1200 \mathrm{MW}_{\mathrm{e}}$ pressurized water reactor temperature and water volume transients after double-ended main cold leg break.

These tests have shown that the highest cladding temperatures will occur under the limiting design case. The rupture limit, however, of the fuel rods will not be reached since the rod surface temperatures will remain below the maximum permissible value of about $700^{\circ} \mathrm{C}$.

Finally, these experiments and calculations have confirmed that the safety injection system of the SIEMENS PWR prevents appreciable damage to the fuel rods, even under the extreme conditions of the "maximum credible accident". The system thus greatly limits the emission of radioactivity in to the containment atmosphere.

Therefore the scepticism regarding the effectiveness of the core emergency cooling system, as voiced by some new media, is not justified for the design here described.

\section{Problems related to the containment structure}

There is a number of other development problems which are related to structural mechanics. Only a few select questions should be mentioned yet that have greatly influenced the reactor design in the recent past.

More precise models have evolved for calculating the forces of the blowdown process and pressure build-up in the different areas of the containment structure during the limiting design case. These data, used in conjunction with the available values on blowdown reaction forces, permitted a better assessment of the loads on the containment structure. This exact knowledge of the accident chronology led to designs which will reliably prevent fragmentation damage to the containment.

Loads, applied to the containment structure from the outside, in addition to internal loads, have demanded the designer's attention on an ever increasing scale. For instance, consideration was given to the possibility of explosions of chemical compounds such as liquid gases mixing with atmospheric air. The containment structure will have to be designed to withdraw external pressure surges as more and more combustible and explosion-sensitive matter is being handled, shipped and stored in highly industralized regions.

Possible aircraft accidents are another problem in this same category. The probability of an aircraft crashing into and consequently destroying essential parts of a nuclear power station is, admittedly, extremely low. Yet there are some specific locations where such an accident cannot be ruled out completely. Any safety analysis for such a site would call 
for a containment structure capable of protecting the primary loop against crashing aircraft components and of safeguarding the residual heat removal capability.

Present-day safety engineering also tends to design for generally higher postulated earthquake intensities. For arriving under these aspects at aseismic structural designs which are economically reasonable, it is necessary to dispose over exact knowledge on the actual building oscillations. Methods for the dynamic computation of elastic structures are suitable instruments. One of the computation models possible for instance makes use of the method of finite elements. The basic idea of this concept is:

1. Separating all structures and their components into elastic elements and massive elements. The two extremes would be weightless elasticities and completely rigid masses.

2. Compiling the equations of motion for the discrete elements.

3. Solving the coupled systems for equations.

The resultant data on displacement and on acceleration forces can be used for accurate calculations of the loads to be expected for the discrete structural components.

\section{Conclusions and outlook}

Obtaining the answers to all these questions calls for high development costs. Extensive detail designs are, in addition, required for each individual nuclear power station project. The return, however, of all these vast expenditures is the constantly increasing safety standard of the nuclear power stations being built.

The safety engineering knowledge and the facilities available allow to build PWR power stations even in municipal locations today. In the years to come a great amount of feedback information from existing plants is to be expected which should lead to further improve the design of reactor components. Moreover, one can safely state already today that a pressurized water reactor with a unit rating of $2000 \mathrm{MW}_{\mathrm{e}}$ and more, i.e. with a unit rating that will be interesting in the eighties, will be feasible even at appreciably lower initial costs. This should well qualify the prognosis that the PWR will retain its place besides the fast breeder reactor owing to its relatively low initial cost. 